\title{
A NOTE ON INTERPOLATION BETWEEN THE ARITHMETIC-GEOMETRIC MEAN AND CAUCHY-SCHWARZ MATRIX NORM INEQUALITIES
}

\section{LIMIN ZOU AND YOUYI JIANG}

Abstract. In this short note, we obtain an inequality for unitarily invariant norms which is a generalization of one shown by Audenaert [Oper. Matrices. 9 (2015) 475-479]. An application of our result is also given.

Mathematics subject classification (2010): 15A60, 47A63.

Keywords and phrases: Unitarily invariant norms, Cauchy-Schwarz inequality, arithmetic-geometric mean inequality.

\section{REFERENCES}

[1] K. M. R. AudenAert, Interpolating between the arithmetic-geometric mean and Cauchy-Schwarz matrix norm inequalities, Oper. Matrices, 9 (2015), 475-479.

[2] R. Bhatia, Matrix Analysis, Springer, New York, 1997.

[3] R. Bhatia, C. DAVIS, More matrix forms of the arithmetic-geometric mean inequality, SIAM J. Matrix Anal. Appl. 14 (1993), 132-136.

[4] R. BhatiA, C. DAVIS, A Cauchy-Schwarz inequality for operators with applications, Linear Algebra Appl. 223/224 (1995), 119-129.

[5] R. Bhatia, F. Kittaneh, On the singular values of a product of operators, SiAM J. Matrix Anal. Appl. 11 (1990), 272-277.

[6] R. Bhatia, F. Kittaneh, Notes on matrix arithmetic-geometric mean inequalities, Linear Algebra Appl. 308 (2000), 203-211.

[7] R. A. Horn, R. Matthis A, Cauchy-Schwarz inequalities associated with positive semidefinite matrices, Linear Algebra Appl. 142 (1990), 63-82. 